
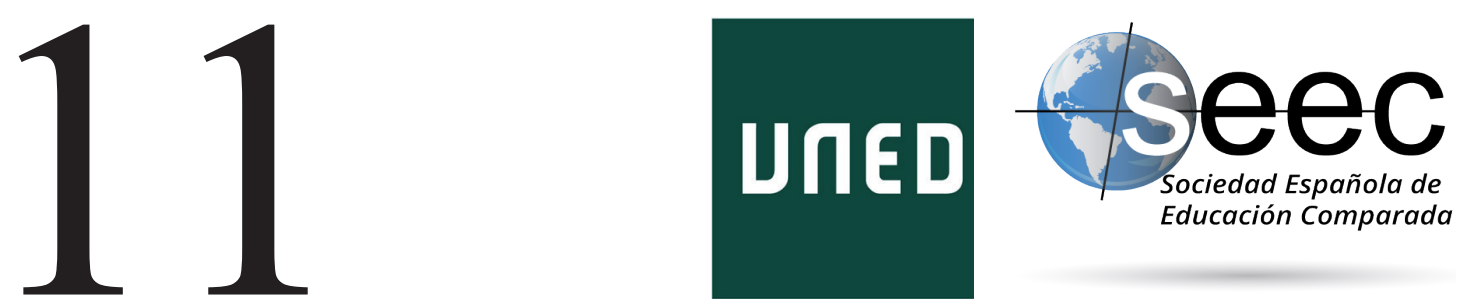

\title{
La mercantilización de la Educación Superior a través del modelo universitario inglés: elementos clave, críticas y posibilidades ${ }^{t}$
}

\author{
The marketisation of Higher Education \\ through the English university model: key \\ elements, criticisms and possibilities
}

\section{David Menéndez Álvarez-Hevia*; Reyes Hernández-Castilla**}

DOI: $10.5944 /$ reec.37.2021.27592

\author{
Recibido: 28 de mayo de 2020 \\ Aceptado: 18 de agosto de 2020
}

\footnotetext{
*David Menéndez Álvarez-Hevia: Diplomado en Magisterio, Licenciado en Pedagogía y Psicopedagogía, Master en Investigación en Ciencias Sociales y Educación. Doctor en Educación. Senior Lecturer en Education Studies en el departamento de Childhood, Youth and Education Studies de Manchester Metropolitan University (Reino Unido). Investigador del Education and Social Research Institute (ESRI) y miembro ejecutivo de British Education Studies Association (BESA). Datos de contacto: Manchester Metropolitan Univeristy Faculty of Education (Brooks Building) 53 Bonsall Street, Manchester M15 6GX; e-mail: d.menendez-alvarez-hevia@mmu.ac.uk

**Reyes Hernández-Castilla: Licenciada en Pedagogía y Psicopedagogía, Doctora en Pedagogía. Profesora Titular en la Universidad Autónoma de Madrid. Miembro del grupo de investigación «Cambio Educativo para la Justicia Social» GICE y la Cátedra UNESCO en Educación para la Justicia Social de la UAM. Datos de contacto: Universidad Autónoma de Madrid de Madrid, Facultad de Formación de Profesorado y Educación, Campus de Cantoblanco, Avenida de Francisco Tomás y Valiente, 28049, Madrid; e-mail: reyes. hernandez@uam.es
} 


\title{
Resumen
}

Este artículo aporta una reflexión teórica sobre los retos asociados a las prácticas mercantilistas y la tendencia economicista de la educación superior. Se toma como referencia la experiencia universitaria inglesa para ilustrar cómo la disposición mercantilista transforma las relaciones entre participantes, funciones, organización y la forma de entender la educación superior. Para ello, el artículo se centra en tres elementos fundamentales que sirven para explicar el proceso de mercantilización de la educación superior inglesa. Primero, la transformación del estudiante en consumidor y la educación en un producto de mercado. Segundo, el concepto de empleabilidad, considerando sus implicaciones pedagógicas y la forma en que promueve concepciones instrumentalistas de la educación universitaria. Tercero, las prácticas asociadas a la evaluación e intensificación de la competitividad que se materializa a través de los sistemas utilizados para evaluar la actividad educativa e investigadora. Se proporciona un análisis crítico de estos tres elementos y se discuten ideas para reconfigurar la transformación mercantilista. Como alternativa a su negación, se propone una reorientación a partir de lo aprendido con la experiencia inglesa para mitigar los aspectos más perniciosos de una tendencia, en auge, en los modelos universitarios europeos.

Palabras clave: mercantilización; empleabilidad; universidad; educación superior inglesa; plan Bolonia; supranacional.

\begin{abstract}
This article provides a theoretical reflection on the challenges associated to the practices of marketisation and the economising trend of higher education. We refer to the English university experience to illustrate how the tendency to marketisation transforms relationships between agents, functions, organisation and the form it is understood higher education. The article focuses on three fundamental elements fundamental elements that explain the marketisation of the English higher education. Firstly, we discuss the transformation of the student into consumer and the higher education into a commodity. Secondly, we explore the concept of employability, focusing on pedagogical implications and showing how it promotes instrumentalised conceptualisations of university education. Thirdly, we discuss practices associated with evaluation and enhancement of competition by taking as a reference the systems used to assess teaching and research activity. We provide a critical analysis of these three elements and discuss ideas to reconfigure the transformation caused by the process of marketisation. Besides critical arguments, this article also provides forms to reconfigure practices associated to marketisation. Rather than rejecting marketisation, we suggest a reorientation that eases the most pernicious effects of this trend that is already present in the European university models.
\end{abstract}

Keywords: marketisation; employability; university; English higher education; Bologna process; supranational.

$+\quad$ Proyecto I+d+i Ref. EDU2017-826288-P. La democracia en las escuelas como fundamento de una educación para la Justicia Social. Cátedra UNESCO en Educación para la Justicia Social. 


\section{Introducción}

El vínculo entre educación superior y crecimiento económico es un elemento fundamental para entender la realidad de las universidades actuales, así como los diferentes cambios en los que estas se encuentran inmersas. Crece el número de instituciones de educación superior a nivel global, así como el número de estudiantes y su diversidad, dando pie a la formación de una comunidad universitaria que Brunner (2012) describe como 'abierta, revuelta, heteróclita, ruidosa, no elitista, a la vez burocrática, mercantil e individualizada, propia de la sociedad civil capitalista contemporánea' (p.34). En otras palabras, nos encontramos con más universidades, más estudiantes, más diferencias entre estudiantes e instituciones y nuevas demandas y formas de abordarlas. Los grandes problemas modernos han sido el surgimiento de la profesión académica, la masificación de la universidad, su burocratización anárquica y la politización de la institución (Brunner ,2012). Problemas que ya en el siglo pasado ciertamente asociaba con la universidad Ortega y Gasset (1976) y que parecen haberse extendido e intensificado en este siglo.

Entre los retos que se presentan, están el conflicto de valores: libertad vs excelencia, excelencia $v s$ igualdad, selectividad $v s$ autonomía. En definitiva, se plantea, por lo tanto, un desafío importante para la universidad del siglo XXI que, para dar respuesta a las demandas sociales y económicas de un mundo globalizado y de tendencia neoliberal, se ve sumida en una reconfiguración de sus elementos identitarios y organizativos. Se trata de un movimiento transnacional que acerca cada vez más la educación superior a un discurso economicista vinculado a una lógica de mercado. Para Brown (2015) esto es entendido como una evolución propia de las sociedades capitalistas y neoliberales en las que operan y se constituyen, no solo las universidades, sino todas nuestras instituciones. A pesar de que se trata de una tendencia global, se entiende que no todos los sistemas universitarios afrontan estos retos de la misma forma o al mismo ritmo. Los diferentes contextos sociopolíticos y económicos, así como las diferentes tradiciones universitarias, condicionan los tiempos y la implementación de los cambios (Neave, 2001).

En este artículo nos apoyamos sobre el modelo universitario inglés para discutir el fenómeno de la mercantilización y sus implicaciones. Para ello explicamos y analizamos las tensiones asociadas a las tres ideas clave que caracterizan la mercantilización de las universidades inglesas. Cada una de estas ideas emerge de los fundamentos del discurso mercantilista destacado en la literatura. Tomamos este modelo especifico como referencia por dos razones. Primero, porque vemos en este que la tendencia mercantilista está más asimilada e instaurada que en otros sistemas europeos. Y segundo, porque existe una gran influencia del modelo anglosajón en la propuesta transformadora del espacio europeo de educación superior propuesto en el Plan Bolonia (Salaburu, Haug y Mora, 2011), con similitudes tanto en enfoques y practicas pedagógicas, como características organizativas e ideológicas (González-Serrano, 2011; De Faramiñan Gilbert, 2010). Teniendo en cuenta esta consideración, podemos afirmar- aunque con cautela-que existe cierta relación entre el modelo inglés y el resto de los modelos europeos. Como mencionamos previamente, el modelo inglés se caracteriza por encontrarse en un estadio del proceso de mercantilización más avanzado y esto nos permite mirarlo desde cierta distancia, pero enfocándolo como una hipotética proyección de futuro hacia la que se pueden desplazar otros sistemas.

Pretendemos aportar una reflexión sobre la política supranacional entendida desde la conjunción de las políticas educativas de los países miembros de la Unión Europea en su proceso de mercantilización con una perspectiva internacional en sí misma y en 
la comparación de estas políticas. La referencia al sistema de educación superior inglés se plantea como paradigma inspirador de políticas supranacionales como la de la propia Unión Europea. Ha sido un Estado miembro de la Unión Europea, y el rastro que ha dejado es más que patente, aunque su presencia siempre ha sido distante y peculiar, más allá incluso del propio proceso del Brexit.

Las reformas de los sistemas de educación superior no han brotado solamente motivadas por mantener su tradición investigadora o docente, sino que plantean como objetivo preparar a la región europea para ser en una zona competitiva, y rivalizar con los Estados Unidos en el mercado de la educación (Sanz-Fernández, 2006). No hay que olvidar que la Unión, desde su nacimiento está vinculada a un acuerdo de desarrollo económico. ${ }^{1}$

Europa ha movilizado a sus instituciones superiores, no desde lo político ni lo científico; sino desde las demandas del mercado laboral (Pardo, 2013). La libre circulación de ciudadanos, y trabajadores, reclama urgentemente el reconocimiento mutuo de títulos profesionales, lo que ha impulsado convenios que así lo permitieran como el tratado de Lisboa de 1997, firmado por la UNESCO y el Consejo de Europa. Este hecho finalmente llevó a la construcción del Espacio Europeo de Educación Superior (EEES), nacido en el proceso de Bolonia, de 1999. Este espacio se organizó en primer lugar, a través del Sistema Europeo de Transferencia de Créditos (ECTS, siglas inglesas), y, en segundo lugar, mediante el diseño de las titulaciones, basándose en un modelo de competencias (profesionales) que se adecúen al mercado laboral. Las ideologías, los valores, y las dinámicas subyacentes en el diseño de las políticas dentro de la Unión (Valle, 2004) hacen recomendable una reflexión sobre el sistema de educación superior británico, que se lleva años implementado, y nos permite recapacitar sobre la evolución y consecuencias de dicho modelo, además de cómo ha permeado en la política de educación superior en la propia Unión Europea.

Las universidades inglesas gozan de una gran reputación y atención global, ya sea por su tradición educativa cuyo comienzo algunos autores sitúan en la Edad Media (Collini, 2012) o, más recientemente, por su fuerte presencia en los rankings internacionales, que año tras año sitúan a sus instituciones en lo más alto basándose en su producción (ver Tabla 1: Presencia de universidades inglesas en los rankings mundiales de educación superior).

Tabla 1.

Presencia de universidades inalesas en los rankinas mundiales de educación superior

\begin{tabular}{|c|l|l|}
\hline Posición & \multicolumn{1}{|c|}{ QS World University Rankings 2020 } & Times Higher Education World University Rankings 2020 \\
\hline 1 & Massachusetts Institute of Technology (Estados Unidos) & University of Oxford (Reino Unido) \\
\hline 2 & Stanford University (Estados Unidos) & California Institute of Technology (Estados Unidos) \\
\hline 3 & Harvard University (Estados Unidos) & University of Cambridge (Reino Unido) \\
\hline 4 & University of Oxford (Reino Unido) & Stanford University (Estados Unidos) \\
\hline 5 & California Institute of Technology (Estados Unidos) & Massachusetts Institute of Technology (Estados Unidos) \\
\hline 6 & ETH Zurich - Swiss Federal Institute of Technology (Suiza) & Princeton University (Estados Unidos) \\
\hline 7 & University of Cambridge (Reino Unido) & Harvard University (Estados Unidos) \\
\hline 8 & University College London (Reino Unido) & Yale University (Estados Unidos) \\
\hline 9 & Imperial College London (Reino Unido) & University of Chicago (Estados Unidos) \\
\hline 10 & University of Chicago (Estados Unidos) & Imperial College London (Reino Unido) \\
\hline
\end{tabular}

Fuente: Elaboración a partir de QS y THE 2020 rankings

1 Su origen se encuentra en la Comisión Europea del Carbón y del Acero, la CECA de 1950, pasó a ser la Comunidad Económica Europea, y de ahí a la Unión Europea. 
Estos rankings supuestamente basados en una medición de la calidad presentan cuestiones profundas. La noción de calidad, que es un concepto relativo, plantea ya desde su base un problema (Gautier, 2007). El problema de plantear la «calidad educativa utilitarista» que de manera implícita incluye la medición, la adición, o la maximización, así como las demandas exógenas que adquieren un rango de norma en los diferentes sistemas educativos. La complejidad de los sistemas hizo que el propio concepto se convirtiera en impreciso y confuso (Cuenca, 2012) aunque provocó fructuosos e infructuosos esfuerzos por precisarlo (Dussel, 2007). La mirada utilitarista promovió reformas neoliberales en los años noventa, concibiendo la educación como un proceso igual para todos, no importa en qué contexto ni qué características tienen, ni siquiera quiénes participan de él. Como reacción a esta idea de calidad, en base a criterios basados en los resultados, emergieron otras perspectivas más humanistas. De manera que los enfoques interculturales y de derechos se transformaron en marcos de referencia alternativos, al poner su acento en las personas que son las depositarias del derecho a la educación. Sin embargo, con un impacto en la economía doméstica de 95 billones de libras, que conlleva el 1,2 \% del Producto Interior Bruto (Oxford Economics, 2017), no es de extrañar que el espacio de educación superior inglés trascienda lo meramente académico o educativo, pasando a ser un sector estratégico de alto interés para la economía nacional. La asociación entre educación superior y crecimiento económico es inevitable, pero requiere de la implementación de diferentes políticas de consolidación y desarrollo (Willetts, 2017). Sin embargo, este interés planteado desde una perspectiva más economicista que educativa o social, conlleva iniciativas que introducen nuevas dinámicas y una lógica mercantilista, en conflicto con visiones más humanistas o Humboldtianas (Docherty, 2011).

Se presentan, de esta manera, cambios organizativos y pedagógicos que, a su vez, operan a un nivel más subjetivo. Se llega a replantear la identidad de los agentes educativos, así como el valor y propósito de la educación universitaria. Autores como Ball (2012) y Collini (2012) explican que la universidad inglesa ha pasado de una concepción de universidad sustentada sobre un equilibrio entre el desarrollo social y público-como refleja el Robbins Report (Robbins, 1963) -, a un sistema dominado por dinámicas mercantilistas y consumistas, que poco a poco han pasado a ser los elementos constitutivos de la concepción moderna. Ante esta tendencia, no faltan las voces críticas en el mundo académico (por ejemplo, Collini, 2017; Docherty, 2015; Giroux, 2014; McGettigan, 2013) desde las que se comparte una oposición hacia los planteamientos mercantilistas. Autores como Harvey (2005) y Brown (2015) enfatizan la idea de que la concepción actual de universidad difícilmente puede entenderse sin ser relacionada con la ideología neoliberal que da sentido a las políticas del mundo globalizado.

Para definir cómo el discurso mercantilista se vincula a la educación superior, partimos de tres ideas o elementos fundamentales que emergen de la literatura anglosajona:

1. Prácticas de gestión propias de sectores comerciales, así como la proliferación de relaciones de mercado (Kolsaker, 2008; Bryson, 2004; Meyer, 2002).

2. Transformación de los profesores e investigadores universitarios en capital humano, de los estudiantes en consumidores, y de la formación universitaria junto al conocimiento en productos del mercado (Tomlinson, 2017; Williams, 2013; Molesworth, Nixon y Scullion, 2009), contribuyendo a la asimilación de planteamientos utilitaristas e instrumentales sobre el valor de la educación superior (Brown, 2015; Giroux, 2014). 
3. Aceptación de una cultura de la competición y de la rendición de cuentas basada en técnicas de control, medición y diagnóstico, tanto en el ámbito de la docencia, como en el de la investigación (Feldman y Sandoval, 2018; Thiel, 2018; Olssen, 2016; Biesta, 2016).

Estos fundamentos se materializan en diferentes prácticas y rutinas que regulan y constituyen el funcionamiento de las diferentes instituciones universitarias inglesas, así como las actividades y concepciones de los agentes educativos vinculados a ellas. Este artículo aborda críticamente las dinámicas mercantilistas en el espacio de educación superior inglés que influyen sobre el modo de concebir la universidad, en cuanto a sus propósitos y la relación entre sus miembros. Invitamos a la reflexión crítica sobre este sistema y sus consecuencias, así como la transferencia de este modelo sin considerar los efectos de su aplicación que ya son visibles en el sistema universitario inglés. Empezamos centrándonos en el primer fundamento, fijándonos en la transformación del estudiante en consumidor, el incremento de las tasas universitarias y el interés por la satisfacción del estudiante. En segundo lugar, exploramos la forma en la que el concepto de empleabilidad influye el discurso pedagógico, correspondiéndose con el segundo fundamento. En tercer lugar, en línea con el tercer fundamento, reflexionamos sobre las iniciativas de evaluación docente e investigadora. En la última parte del artículo se profundiza sobre algunas de las críticas, pero también se exploran nuevas posibilidades asociadas a los cambios que se proponen desde el planteamiento mercantilista.

\section{La transformación del estudiante en consumidor}

Un momento clave para entender el proceso de mercantilización de la educación superior inglesa y cómo se ha ido intensificado en los últimos años, fue la publicación del conocido informe Browne (Browne, 2010) y la aprobación del Libro Blanco de la educación superior (DfBIS, 2011). Ello conllevó, entre otros cambios, a la introducción en el curso académico 2012-2013 de un nuevo régimen de financiación universitaria que despojó a las universidades de gran parte de su financiación estatal. Como consecuencia, el precio de las matrículas universitarias aumentó hasta situarse entre las más altas del continente europeo. Esto también vino acompañado de nuevas condiciones en el sistema de prestamos para pagar los estudios, lo cual no supuso una merma en el número de estudiantes ya que siguió en una tendencia al alza como se muestra en el siguiente gráfico [figura 2: Crecimiento del número de Estudiantes universitarios en Reino Unido 2014-2019]: 


\section{Estudiantes Universitarios en Reino Unido}

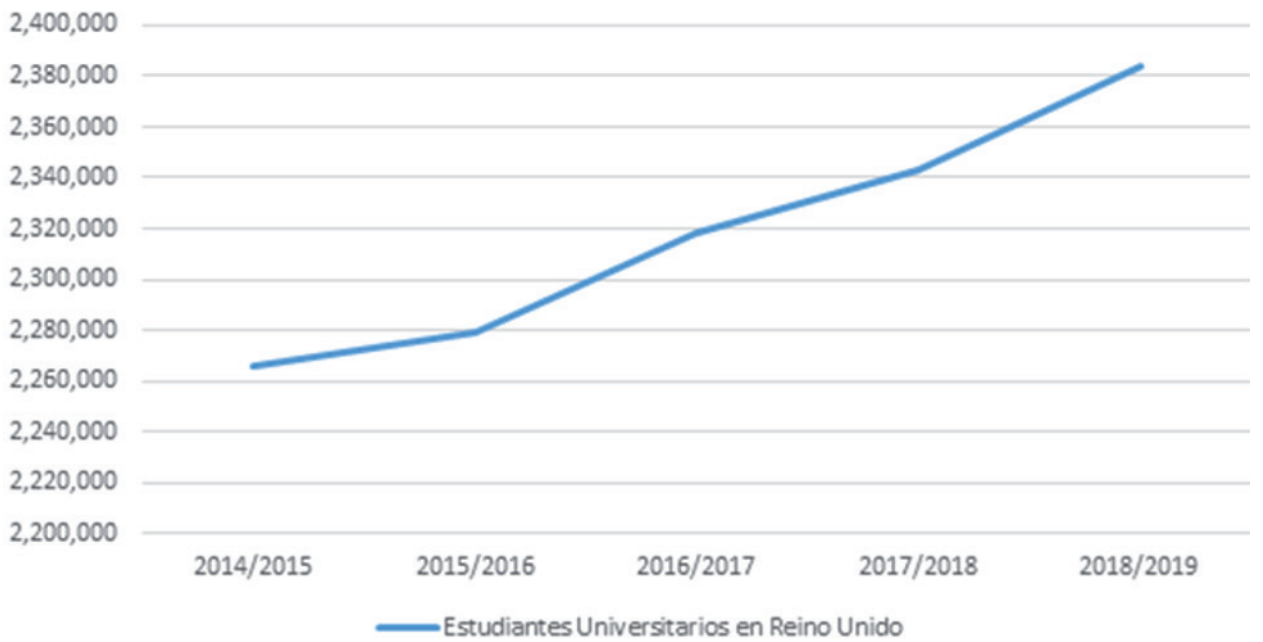

Figura 1: Crecimiento del número de estudiantes universitarios en Reino Unido 2014-2019

Fuente: Elaboración propia. Fuente: Higher Education Statistics Agency https://www.hesa. ac.uk/data-and-analysis/sb255/figure-3)

Los cambios en la política de financiación universitaria han afectado a las relaciones educativas y en especial al comportamiento de los estudiantes (Bates y Kaye, 2014; Jones, 2010). Para Williams (2013), al igual que para Brown (2015), el incremento de las tasas en conjunción con otros cambios políticos, históricos y culturales, han acabado por convertir a los estudiantes en consumidores. Sirva como ejemplo la Consumer Right Act, es decir, la Ley de los Derechos del Consumidor, 2015, en la que se reconoce la posibilidad de presentar reclamaciones si el estudiante no está satisfecho con los servicios recibidos en la Universidad.

En este contexto, se facilita que la identidad del estudiante se solape con la identidad del consumidor, y se legitimiza y normaliza la interpretación de la experiencia educativa dentro de una lógica de consumo o economizada. Se establece así una relación de clienteproducto-proveedor que sitúa a sus participantes dentro de un discurso económico que, trasladado a un contexto como el educativo, puede ocasionar la alteración de los modos de proceder de los agentes educativos, sus expectativas y las dinámicas institucionales. En esta lógica, el cliente es el estudiante que paga por recibir una formación. Su educación es el producto y el proveedor es la institución educativa, que se personaliza en el profesor.

La satisfacción del cliente o consumidor (estudiante) con el producto adquirido y/o servicio recibido (educación) se vincula con el precio pagado. En esta fórmula, el proceso educativo pasa a ser una mera transacción y el estudiante puede quedar atrapado en lo que Tomlinson (2018) describe como 'la trampa del valor-por-dinero' (p.725, traducción propia) que le lleva a evaluar su experiencia educativa en términos economicistas que relacionan calidad/precio, reduciendo su capacidad para desarrollar un análisis más profundo de su posición y el proceso educativo.

La transformación de los estudiantes en consumidores se hace especialmente perceptible a través del cambio en sus expectativas y en su capacidad para influenciar el funcionamiento del mercado educativo (Barnett, 2011). En la práctica, nos encontramos con dinámicas en las universidades que van encaminadas a satisfacer antes sus demandas 
como consumidores que como estudiantes. Por ejemplo, con inversiones millonarias en la construcción de nuevos edificios de vanguardia que cuenten con la última tecnología, mientras que se observan casos en los que elementos tradicionalmente asociados a calidad educativa, como son las horas de atención al estudiante, de clases presenciales o la ratio estudiante-profesor se ven alteradas negativamente. Por otro lado, el fenómeno de la «inflación» de notas nos proporciona otro ejemplo ilustrativo de este cambio. Para Bachan (2017), es evidente y llamativo el incremento observado en los últimos años de estudiantes que adquieren resultados excelentes. Esto es difícilmente justificable únicamente desde el argumento que plantea una mejora en el talento de los estudiantes o la enseñanza, y de ahí la sospecha de que se trate de una respuesta institucional a las exigencias y presiones para atraer, mantener y satisfacer al estudiante-consumidor. Paradójicamente, estas dinámicas para satisfacer al estudiante-consumidor pueden tener un impacto negativo cuando se prioriza el proteger y reforzar el valor de la institución, dentro de un mercado altamente competitivo, frente a su desarrollo académico y profesional.

\section{El Foco sobre la empleabilidad}

En estos últimos años hemos visto cómo ha pasado a ser un elemento de especial interés la capacidad de las universidades para que sus egresados se integren en el mercado laboral. Esto ha conllevado múltiples transformaciones, tanto a nivel pedagógico y organizativo, como ideológico (Artess, Hooley y Mellors-Bourne, 2017). Se trata de un fenómeno sobre el que, al igual que en otros países, el gobierno del Reino Unido viene reclamando más atención (DfES, 2003; Tomlinson 2012). Para comprenderlo más profundamente debemos de fijarnos en el concepto de empleabilidad, que se define como el potencial de los egresados universitarios para obtener y mantener un trabajo que corresponda con su cualificación (Yorke, 2006; Knight y Yorke, 2003). Este concepto está muy presente en la publicitación de cualquier universidad inglesa y juega un papel fundamental a la hora de crear métricas que evalúan el rendimiento institucional.

Desde el punto de vista pedagógico, Knight y Yorke (2004) destacan las siguientes cuestiones asociadas al desarrollo de la empleabilidad en las universidades inglesas:

- Oferta de prácticas profesionales diversas como parte de los planes de estudio.

- Flexibilidad en los planes, que permita adaptarlos a las demandas cambiantes del mercado laboral.

- Enfoque didáctico que conecte el aula con el mundo laboral por medio de planteamientos pedagógicos como el aprendizaje por proyectos o por competencias.

- Desarrollo de asignaturas o unidades didácticas centradas específicamente en el desarrollo y gestión de habilidades profesionales.

- Acreditación de habilidades profesionales alcanzadas durante los estudios.

- Fomento de una actitud emprendedora y empresarial

- Aprovechamiento de las habilidades adquiridas en trabajos que no requieren cualificación o de voluntariado.

- Extensión de servicios especializados en asesoramiento, orientación y seguimiento laboral. 
Los cambios políticos de los últimos años y las prácticas que estos han introducido en el mundo universitario inglés, lo han situado en conceptualizaciones de empleabilidad asociadas a ideas individualistas centradas en el desarrollo de habilidades y capacidades. Para Boden y Nedeva (2010), la transformación que conlleva el discurso de empleabilidad adoptado por las universidades inglesas se explica en tres puntos. Primero, en la división entre instituciones de educación superior que forman a líderes e instituciones de segunda clase que se encargan de formar trabajadores dóciles. Segundo, las universidades ya no solo tienen que responder a las demandas sociales o del gobierno, ahora también responden directamente a las demandas externas del sector industrial y empresarial. Todos ellos cada vez con más fuerza y llegando a influir sobre el currículum. Finalmente, el tercer punto, el efecto de promover unos valores individualistas basados en la lógica del mercado y las posibilidades laborales individuales. Con esto se deja en un segundo plano un enfoque más crítico del mercado laboral y las posibilidades colectivas para transformarlo y hacerlo más equitativo y justo. Se restringe el potencial de trabajo colectivo, a través del cual profesores y estudiantes construyen alternativas que pueden servir como ideas que guíen nuevos proyectos de transformación política y social (Noonan y Coral, 2015).

Los argumentos expuestos hasta ahora podrían resumirse diciendo que las universidades inglesas están reorientándose para dar respuestas a las voces que reclaman una educación que prepare directamente para el mundo laboral. Se trata en parte de una respuesta centrada en la oferta de servicios y cambios pedagógicos, que buscan satisfacer las demandas de estudiantes, gobernantes y sector empresarial, seguramente con un orden de prioridades inverso al expuesto. Sin embargo, consiste en un cambio que viene de la mano de una forma de entender el propósito de la educación superior desde un discurso utilitarista y que entra en conflicto con otras formas más holísticas de concebirla.

\section{Midiendo docencia e investigación}

Una de las características principales de la educación superior inglesa es que tiene normalizada una cultura de evaluación y auditoría (en la literatura inglesa aparece como accountability practices), que se ha visto especialmente intensificada en los últimos 25 años con la creación de diferentes organismos de control de calidad como, por ejemplo, la Quality Assurance Agency (Agencia Garante de Calidad) y la Office for Students (Oficina para el Estudiante). Esto viene de la mano de intereses políticos y socioeconómicos que demandan una rendición de cuentas a través de la cual interpretar resultados para informar y justificar decisiones, tanto administrativas (e.g. distribución de recursos), como docentes o del estudiante (e.g. selección de universidad en la que estudiar o trabajar).

Estas prácticas de auditoría se justifican a través de la lógica de mercado. El consumidor demanda información detallada y rigurosa para que pueda tomar decisiones-libres e informadas-sobre el producto (Jongbloed, 2003). Se espera que el estudiante haga uso de estos datos para seleccionar la universidad en la que va a estudiar, teniendo en cuenta que el precio a pagar será aproximadamente de 9,00o£ al año, pudiendo ser el doble si el estudiante no es ni británico ni europeo. El precio es independiente de la universidad o carrera estudiada. Un elemento fundamental de este planteamiento es el rigor, la validez y fiabilidad de la información a la que acceden los diferentes agentes educativos. Ello requiere un esfuerzo por crear un sistema de indicadores y métodos de obtención de datos que sirvan verdaderamente para sistematizar, organizar y comparar los datos del sistema universitario con garantías y que permita, a su vez, mejorar la competitividad. 
Las universidades, aunque en diferentes medidas, se centran en dos tipos de actividades: docencia e investigación. Tradicionalmente la investigación ha sido el indicador principal de calidad. Esto es lo que ha diferenciado a universidades del grupo Russelllas más prestigiosas-del resto de universidades, más bien orientadas hacia la educación de masas.

El Research Excellent Framework (REF) es el sistema utilizado desde 2014 para evaluar el impacto y la calidad de la investigación científica en las instituciones de educación superior del Reino Unido. Su antecesor es el Research Assessment Excercise (RAE), que funcionó entre 1986 y 2008, sentando las bases del sistema actual. El REF trajo como una de sus principales innovaciones el que se considerara el impacto del trabajo de investigación más allá de la comunidad científica para justificar su producción en la forma de casos de impacto. Se basa en un sistema de evaluación por pares a través de paneles de expertos, que pueden incluir personal externo al mundo académico. Se dividen en 34 unidades de evaluación que son, a su vez, supervisados por 4 paneles principales. El análisis bibliométrico de las publicaciones no es determinante. Este tiene un peso relativo, siendo utilizado como soporte adicional a la decisión de los expertos. Dicha evaluación tiene lugar aproximadamente cada 5 años y sus resultados se presentan en 5 categorías que están entre líder mundial $\left(4^{*}\right)$ a sin clasificar o sin relevancia científica ( $0^{*}$ ). Cada institución es responsable de seleccionar los trabajos que van a ser evaluados y asociarlos a un panel de evaluación. Se consideran publicaciones, casos de impacto de investigación e incluso la financiación externa obtenida. El resultado final de la evaluación permite crear rankings interuniversitarios y facilita las comparaciones entre departamentos o disciplinas dentro de cada universidad. Una evaluación positiva de trabajos individuales dentro de las categorías superiores determinará la financiación pública que recibirá la institución. En otras palabras, el REF es una herramienta administrativa para justificar diferentes asignaciones de fondos públicos destinados a la investigación desde las universidades. La mayor parte de la financiación para investigación de la universidad se determina a través de los ingresos derivados de él y, sobre todo, a través de los beneficios generados por contratos de investigación en concurso público.

Por otro lado, la experiencia educativa de los estudiantes y en especial, el cómo está asociada a la actividad docente, ha recibido especial atención en los últimos años. Cada institución desarrolla sus propias herramientas internas para evaluar asignaturas, programas o prácticas. A nivel nacional, el National Student Survey (NSS) ha sido hasta el momento la principal fuente de información sobre la opinión de los estudiantes con respecto a su desarrollo y experiencia en la universidad. Se trata de un cuestionario anónimo con 27 preguntas que es completado cada año por estudiantes de grado durante su último año académico. Los resultados son públicos y buscan proporcionar información sobre la calidad educativa de las distintas universidades, para, de esta forma, ayudar a posibles estudiantes en la elección de universidad. No obstante, el NSS no está exento de críticas que cuestionan la capacidad para evaluar la satisfacción y calidad (Brown, et al., 2015; Pickford, 2013), así como el uso de los resultados para la toma de decisiones (Gibbons, Neumayer y Perkins, 2015; Thiel, 2018). En 2015, el gobierno empezó a desarrollar el Teaching Excellence and Student Outcomes Framework (TEF) que ha sido implementado de forma creciente, y que se encuentra en su última fase de adaptación (DfE, 2017). A través del TEF se propone una forma más sofisticada e innovadora de evaluar la calidad de la enseñanza en la educación superior. En la práctica incorpora en su esquema la información del NSS, pero también aporta datos sobre empleabilidad, 
calificaciones de entrada, progresión y características de los estudiantes. Esta información es complementada con un informe redactado por cada institución. La evaluación considera las singularidades de cada caso y es llevada a cabo por paneles formados por estudiantes, académicos y otros expertos. La polémica ha emergido ante la propuesta de que el resultado obtenido en futuros TEF pueda ser esgrimido como argumento para que algunas universidades lo puedan vincular al incremento en el precio de sus matrículas universitarias (THE, 2016). El TEF se presenta como parte integral de un amplio conjunto de reformas que están diseñadas para mejorar la competitividad y la elección en la educación superior, aumentando la transparencia y dotando de mayor flexibilidad al sistema (Gunn, 2018). También significa una reivindicación de la actividad docente en la universidad, generalmente deslucida por la labor investigadora aun cuando es sabido que la primera es la principal fuente de ingresos. Por otra parte, el TEF también contribuye a la afirmación del estudiante como consumidor y de la educación universitaria como producto.

\section{Criticas y posibilidades}

Los elementos claves presentados en las secciones previas caracterizan la modernización de las universidades inglesas. Una modernización que conlleva principalmente cambios relacionales, de organización y gestión, de financiación y de justificación. Estas transformaciones han sido orientadas para adaptar un sistema universitario en constante crecimiento a una sociedad en la que impera una lógica de mercado que subyace a la ideología neoliberal (Maisuria y Col, 2017). Se busca convertirlo en un sistema sostenible y competitivo, capaz de responder a las demandas sociales, pero que principalmente pretende ajustarlo a una economía de mercado.

En lo que se refiere a la Unión Europea, una de las políticas educativas con mayor tradición es la política universitaria, porque se halla enmarcada dentro de la formación profesional, o de profesionales. Una economía de mercado exige el reconocimiento de los títulos y diplomas universitarios, para ello es esencial que pueda haber un intercambio entre docentes y estudiantes, facilitado mediante los programas ERASMUS, y potenciando el vínculo entre universidades y empresas. Sin embargo, podemos señalar que la línea de actuación más fuerte es la de la Formación Profesional, precisamente por la demanda original de la Comunidad Económica Europa de formar profesionales que favorecieran la transición a la vida activa en el mercado laboral (Egido, 1995).

Ante estas dinámicas de cambio, cabe destacar una resistencia interna que se ha intensificado en los últimos 10 años y que va más allá de las fronteras británicas o europeas, uniendo a estudiantes y académicos a nivel global, por ejemplo, mediante los mencionados programas de intercambio. Hemos visto en el Reino Unido tanto a estudiantes como profesores de universidad manifestarse en los últimos años contra los diferentes cambios en la financiación de la educación superior, condiciones laborales o herramientas de evaluación. En Estados Unidos, el movimiento Occupy, o en España el 15-M, también contenían proclamas que se asociaban a una oposición hacia políticas educativas que afectaban al devenir de la educación superior, una herencia en este último del movimiento estudiantil anti-Bolonia que precisamente, mostraba el descontento ante una reforma señalada como mercantilizadora (Cilleros y Betancor, 2014). Como ejemplo más reciente esta el movimiento estudiantil de Chile, desde el que se critica un modelo de universidad, y educación en general, con un claro corte mercantilista. No han faltado 
tampoco académicos críticos que han contribuido a cuestionar el impacto del discurso mercantilista y sus prácticas en la forma de entender y funcionar la universidad. A continuación, se profundiza en alguna de estas críticas a la vez que se sugieren formas de afrontar esas propuestas mercantilistas de forma alternativa.

\subsection{Del estudiante como consumidor al estudiante en asociación}

El tema del estudiante como consumidor es un asunto recurrente en la literatura crítica, aunque hay que tener en cuenta que el desplazamiento de la identidad del estudiante hacia la de consumidor es difícil de trazar, con muchas variables externas y, por tanto, de compleja generalización. Esta identidad no es asumida de la misma forma por parte de todos los estudiantes. No podemos ignorar que también existen formas de resistencia a esta tendencia. Hay estudiantes que quieren y reclaman ser estudiantes, así como, profesores que quieren enseñar antes que vender educación, que se enamoren de ser enseñantes y enamoren a sus estudiantes. Esteban-Bara (2019) aboga por una formación de "fastfood" sino de una cocción a fuego lento, con aprendizajes que fermentan, para formar gentes sólidas en tiempos líquidos. En esta línea, Tight (2013) destaca que la metáfora del «estudiante como consumidor» es cruel, dado que no captura la complejidad de la identidad del estudiante y, por lo tanto, no puede dejar de ser cuestionada y revisada. De ahí que diferentes autores apuesten por un enfoque desde el que se deje de demonizar al estudiante como sujeto pasivo y se busque entender las diferentes formas en las que se manifiesta la resistencia a las dinámicas mercantilistas y las posibilidades que emergen a partir de estas (e.g. Giroux, 2014; Williams, 2013; Molesworth, Nixon y Scullion,2009).

La alternativa que planteamos a este modelo de conceptualizar al estudiante como consumidor pasa por reconfigurar las relaciones entre estudiantes y profesores. Como explica Thiel (2019), las diferentes políticas de mercantilización de la universidad acaban por separar y enfrentar a sus miembros, obstaculizando cualquier tipo de identidad colectiva que va más allá de los intereses como consumidores. Esto crea diferentes tensiones entre grupos y dentro de ellos y, aunque puedan ser percibidas, no son contrarrestadas. Recuperar alianzas y asociaciones entre estudiantes y profesores se presenta, por lo tanto, como una forma de resistencia productiva y factible contra estas políticas. Se trata de reconocer que las presiones por consolidar la cultura consumista no benefician a ninguna de las partes. Se pretende animar la búsqueda de otras alternativas. Esta idea es llevada a la práctica por Neary y Saunders (2016), quienes basándose en los principios y prácticas de la teoría critica, implementaron un modelo de colaboración entre estudiantes y profesores en la universidad de Lincoln. Se fomentó el uso de herramientas de la pedagogía crítica en el aula para analizar la situación de los estudiantes y el profesorado dentro y fuera del contexto universitario. Además, se establecieron formas de participación activa de los estudiantes en niveles a los que su intervención había sido limitada (e.g. en torno a tareas de investigación, gestión de programas y gestión institucional). El resultado es 'un acto de colaboración entre estudiantes y académicos en el proceso de creación de un conocimiento práctico y crítico' (Neary y Saunders, 2016, p. 9, traducción propia) que sirve para afrontar conjuntamente las contradicciones y defectos del sistema mercantilista, e imaginar formas alternativas de articular la identidad como estudiante, profesor universitario o entender la universidad. 


\subsection{De la empleabilidad como meta, a la empleabilidad como posibilidad}

El vínculo directo entre la educación superior y el mundo laboral es fundamental para entender la universidad de hoy día y su futuro. Las universidades inglesas se han centrado en reforzar esta relación hasta el punto de que la formación orientada a lo laboral pasa a ser un elemento central que condiciona considerablemente otras funciones (Noonan y Coral 2015). Autores como Giroux (2014) y Gumport (2000) entre otros, advierten de que una excesiva instrumentalización de la educación superior pone en riesgo algunos de los valores intelectuales, democráticos y culturales que hacen de la universidad un referente social. Afectando también a la forma en que profesores universitarios entienden su función (Morrison, 2014; García-Ruiz, 2011). Por otro lado, hay universidades-en especial las más orientadas a la docencia-que se replantean la relación teoría-práctica, siendo este un debate nuclear para entender la posición específica de la universidad en referencia a otras formas de educación y entender su propedéutica. Esto, supone que disciplinas o programas de estudio que no tienen un fuerte vínculo practico y/o económico se puedan ver perjudicadas, llegando a estar en riesgo de desaparición, y con ellas su contribución a otros aspectos de nuestra sociedad.

El que la empleabilidad sea entendida como un indicador para comparar la calidad educativa es también un aspecto cuestionable en la literatura. Harvey (2001) explica que la empleabilidad como elemento comparativo entre instituciones tiene como efecto el alejar a estas de su contexto local y sus características socioeconómicas más específicas. A ello Glover, Law y Youngman (2002) añaden que puede generar expectativas laborales irreales en los estudiantes, siendo un punto crucial para entender un planteamiento crítico que sirva para contestar las presiones mercantilistas.

$\mathrm{Al}$ plantear críticas e intentar imaginar una alternativa al modelo mercantilista de empleabilidad, no podemos caer en la tentación de negar que el preparar profesionales altamente cualificados es una de las funciones y responsabilidades de la universidad actual. Lo que sí se puede es abrir un debate interno sobre la forma en la que cada institución universitaria entiende su enfoque hacia lo laboral y la forma en la que los recursos y tiempos asociados a la empleabilidad ocupan el espacio dedicado a otros aprendizajes o funciones. Habría que analizar qué elementos y recursos se han reducido o limitado para ganar en empleabilidad y considerar el impacto que esto tiene en la forma de entenderse la educación universitaria como proceso de formación integral.

De un lado, las instituciones universitarias podrían gozar un planteamiento más realista y transparente, desde el que se reconocieran sus limitaciones para operar en un mercado laboral que, según se extrae de los diferentes estudios internacionales se presenta ante los jóvenes como una alternativa incierta, impredecible, volátil, frágil y condicionada por diferencias estructurales (Caroleo et al. 2017). Por otro lado, tanto profesores como estudiantes deben de ser críticos con la idea de que una formación universitaria basada en la práctica profesional prepara mejor para desempeñar un trabajo altamente cualificado. Sin embargo, en un mercado laboral como el actual, la formación excesivamente instrumental focalizada en el presente inmediato, se desmorona ante un futuro cambiante e incierto mientras que una formación integral y creativa, que permite la flexibilidad ante el cambio, parece más prometedora.

Sin mermar la futura empleabilidad de los egresados, cabe la posibilidad de proponer otros modelos alternativos a la mercantilización en la universidad como, por ejemplo, el de «prácticas de estudio». Un modelo que aúna los procesos científicos y políticos y que 
pretende concebir nuevas formas de convivencia con el objetivo de generar respuestas concretas a problemas actuales a través de enfoques orientados a la práctica, tal y como lo entiende la filósofa belga Isabelle Stengers. Estudiar en la universidad abarca mucho más que aprender y esto conlleva una reinterpretación del concepto de estudio (Masschelein, 2017), entendido como «aprender de nuevo» (Schildermans, 2019) mediante las prácticas de estudio llevadas a cabo en contextos formales y no formales (Bárcena, 2019).

\section{5•3. De la medición a la valoración de su impacto}

Si se analiza el propósito, la forma y los efectos de los mecanismos de evaluación de la actividad docente e investigadora, emergen argumentos que nos permiten desarrollar una visión más profunda. Para empezar, nos encontramos con la idea de que tanto REF como TEF buscan evidencias científicas que proporcionen una visión objetiva de la calidad de las actividades educativas y de investigación de las universidades inglesas. Sin embargo, a pesar del esfuerzo por diseñar sistemas que consideren múltiples variables, utilizando diferentes fuentes de información y mediante un proceso sistemático, autores como Wildson et al. (2015) siguen cuestionando la cientificidad de dichas herramientas. Estos argumentan que los conceptos de excelencia, calidad, satisfacción, eficiencia o impacto son centrales a la hora de diseñar dichos mecanismos, a pesar de que sus significados permanecen abiertos, elusivos y controvertidos. Además, no están exentos de una fuerte carga ideológica, y de ahí que sus mediciones sean refutadas.

Por otra parte, la evaluación llevada a cabo mediante paneles de expertos es una práctica muy extendida, aunque está sujeta a fuertes críticas, desde planteamientos positivistas por los elementos subjetivos que implica. Sin embargo, no se puede negar que aporta una contextualización al proceso de evaluación ya que explica sus resultados y proporciona argumentos de mejora institucional.

Aunque TEF y REF evalúan diferentes aspectos de la actividad universitaria, ambos comparten el propósito de fundamentar la toma de decisiones sobre los agentes educativos. Sin embargo, en una visión reduccionista se pueden traducir en meros valores numéricos e incluso en rankings sensacionalistas y manipulables. Estas dinámicas más que aportar transparencia producen opacidad, dado que las instituciones acaban por centrar gran parte de sus esfuerzos en la construcción y mantenimiento de una realidad pre-fabricada (Ball, 2010). Aunque el impacto de los rankings en la toma de decisiones de los estudiantes o administraciones educativas no sea determinante (Broecke, 2012), existe un alto interés por la obtención y exhibición de resultados positivos, necesarios para operar en el sistema universitario actual. Sin duda, condiciona el funcionamiento y practicas institucionales que lleva a «dar valor a lo medible, dejando de medir lo que es de valor» (Klemencic y Ashwin, 2015, citado en Gunn, 2018, p. 140, traducción propia). O, en otras palabras, otorgando mayor valor a la apariencia que a lo que realmente sucede en las universidades. Es de destacar que iniciativas como el REF han supuesto un cambio en las reglas del juego, lo que conlleva la pérdida de integridad intelectual y profesional de los académicos que se han visto obligados a satisfacer demandas externas y a cuestionar su forma de entender la investigación y su finalidad (Chubb y Watermeyer, 2017). A esto, hay que sumarle la vulnerabilidad emocional, intelectual y profesional sentida por los docentes universitarios expuestos al proceso y sus resultados (Watermeyer y Chubb, 2019). Así, los enfoques más críticos siguen desconfiando de un sistema que se centra excesivamente en generar información, sin considerar el impacto de este proceso en los diferentes niveles. 
En los últimos años hemos podido observar un interés global por instaurar y extender sistemas de medición y evaluación en todas las actividades universitarias. Algunos sistemas, al igual que el inglés, emplean medidas relativamente subjetivas (e.g. grado de impacto, servicios ofrecidos etc.), mientras que otros sistemas han apostado por usar indicadores cuantitativos (e.g. factores de impacto de las publicaciones, calificaciones de docencia etc.). Aunque de diferente forma, ambos comparten un clima de competición que hace que las instituciones y sus académicos se vean empujados a optimizar sus esfuerzos para satisfacer los requerimientos evaluables. Como consecuencia, se dejan de lado otros intereses.

En este proceso se ganan referencias de comparación, pero también se limita la capacidad de la universidad para investigar y educar: se vive para los indicadores. No importa el cómo, ni el para qué, se ha de publicar. Importa el índice de impacto, pero no el sentido del texto, y su capacidad de impactar en la sociedad. Y, este solo importa en la medida de su medida. Si se puede medir, importa por las repercusiones de ascenso en la carrera académica, o en emolumentos económicos directos o indirecto que pueden conllevar. Supone, en muchos casos, la conversión de los investigadores, docentes e instituciones universitarias en «mercenarios de la métrica».

Quizás sea necesario reivindicar el carácter formativo de la evaluación y que esté realmente acompañada de medidas de apoyo al profesorado. Medidas que contribuyan a la planificación de sus actividades, y reforzar y trabajar las debilidades observadas, además de establecer medidas que incentiven su profesionalización. Sin duda, la evaluación debe ir acompañada de un plan de desarrollo profesional, que contribuya a la consolidación de plantillas docentes y, como meta final, la mejora de los procesos docentes e investigadores (Aramburuzabala, Hernández-Castilla, y Ángel-Uribe, 2013). Indudablemente, todo ello pasa por una discusión sobre la concepción de profesor que se demanda en la universidad (Tejedor y Jornet, 2008).

Es difícil imaginar una alternativa que permita escapar de la necesidad institucional de rendir cuentas sobre resultados. Especialmente cuando estamos hablando del nivel educativo que requiere de más recursos económicos, ya sean de origen público o privado. Mientras que el modelo inglés ha apostado por una competición interuniversitaria a través de herramientas como el TEF y el REF para mejorar rendimiento y distribuir recursos públicos, el modelo europeo, a pesar de disponer de mecanismos similares, muestra diferentes actitudes y comportamientos ante el establecimiento de dinámicas de competición.

El modelo de financiación público que impera en la gran mayoría de instituciones universitarias europeas quizás sea lo que marque esta diferencia. En tal caso, cabe preguntarse si la reducción o redistribución de este tipo de financiación acabará por empujar a las universidades europeas hacia la misma posición que las inglesas, acelerando así el proceso de mercantilización a nivel supranacional.

\section{Conclusiones}

En este artículo se ha presentado una reflexión teórica sobre la educación superior, acerca de cómo se define en la práctica, y qué supone su transformación y tendencia mercantilista. Además, se ha intentado esbozar diferentes argumentos que emergen de la literatura y que permiten afrontar esta tendencia de una forma más crítica en otros contextos, como, por ejemplo, el europeo. Este, a pesar de compartir con el modelo inglés 
cierta complacencia hacia la lógica mercantilista, marcado en la hoja de ruta de Bolonia, sigue un ritmo y evolución diferente. Un sistema universitario altamente mercantilizado como es el inglés, que ha servido de inspiración al modelo de la educación superior en la Unión Europea. De hecho, el objetivo estratégico, diseñado para la Unión en el Consejo Europeo de Lisboa (2000), buscó una economía basada en el conocimiento, la más competitiva y dinámica del mundo (Valle, 2012). Para lo cual la educación superior pasó a ser una de las prioridades de la Unión, con el fin de dotar a la universidad de una mayor participación en el desarrollo económico y social de la ciudadanía.

El modelo inglés se nos presenta como una proyección de futuro de la que poder aprender para prevenir determinadas consecuencias y construir alternativas. No se busca romper con esta tendencia, sino proponer posibilidades de reconfiguración para dar respuestas más apropiadas, informadas y contextualizadas. El análisis del sistema inglés aporta una mirada profunda y, a la vez, crítica de lo que es y hacia dónde se encamina el sistema. Nos permite reflexionar acerca de lo que pudiera ser adoptar modelos y prácticas sin un sano escepticismo crítico.

Muchas veces nos olvidamos de que la educación superior tiene un precio y de que, además, este siempre es elevado. Una de las diferencias que se dan en el contexto inglés es que, este precio se hace explícito y la responsabilidad de su pago recae totalmente sobre el estudiante. Al ser esta transacción visible, se justifica la introducción de diferentes dinámicas que hacen que se establezca una lógica mercantilista. En ella, el estudiante actúa como cliente que paga por un producto, buscando que este satisfaga sus expectativas. La educación se presenta como un producto a comprar. Y las instituciones universitarias, encarnadas en la figura del docente, son los proveedores y los últimos responsables de la satisfacción del cliente. Los autores más críticos alertan sobre la posibilidad de que esta fórmula acabe por determinar las prácticas docentes, las relaciones entre estudiante y profesor, en definitiva, la forma de entender la educación (superior). Contra esta posibilidad, se propone reconfigurar la relación estudiante-profesor, no como contrarios o antagónicos, sino como una asociación ineludible para entender y transformar las contradicciones y efectos negativos de una relación que, al verse enmarcada por los parámetros consumistas, puede dificultar y distorsionar su potencial educativo.

Asimismo se ha detallado cómo, a través de la idea de empleabilidad, la universidad modifica la forma de articular su misión. Deja su prioridad tradicional: educar ciudadanos, formar personas cultas, científicos a la vez que formaba profesionales. Y, se pasa a orientar gran parte de sus esfuerzos al aspecto profesionalizante.

De la experiencia inglesa destacamos que un exceso de atención sobre la misión profesionalizante contribuye a la conversión de la universidad en una institución pragmática, reproductora de las necesidades del mercado y del consumo. En este contexto, sus señas de identidad pasan a estar fundamentadas en la productividad, la eficacia y en las propias competencias profesionales que en ocasiones pecan de volatilidad (Morrish y Sauntson, 2013). Con ello la universidad corre el riesgo de dejar de lado su papel como trasmisora y desarrolladora de conocimientos, valores y competencias, asentados sobre la capacidad de formación e investigación para la sociedad y los ciudadanos, para convertirse en una mera visión mercantilista, que concibe la educación como promotora de un desarrollo económico, potenciado desde instituciones e intereses puramente desarrollistas y economicistas. Cuando el conocimiento se disocia del bien común, la educación se instrumentaliza, se instala la competitividad, y surge la necesidad de utilizar otros parámetros para hablar de educación superior de calidad, como son la empleabilidad, la 
competitividad e, incluso, la rentabilidad. ¿Queremos realmente una educación superior altamente instrumentalizada y asentada sobre las máximas neoliberales de competitividad y productividad? Siendo realistas, difícilmente podremos escapar a este énfasis instrumentalista y de ahí que cada vez esté más presente en cualquier modelo universitario. Lo que sí podemos hacer es revindicar que el concepto de empleabilidad no sea entendido de la misma forma y que su uso como indicador de calidad educativa a la hora de crear comparaciones sea abordado de una forma más crítica y contextualizada.

El REF y el TEF son destacados en este artículo como estrategias de evaluación y medición sobre las que se apoya la competitividad en el contexto universitario inglés. Pero chasta qué punto son fiables las estrategias de evaluación y medición que disponemos? ¿Cómo hacemos para que el énfasis en los resultados y en la competitividad no acabe por distorsionar la atención de todas las actividades de la universidad? ¿Qué sucede con aquellas variables que quedan excluidas de estas métricas? ¿Qué ha llevado a dar más peso a unas y a excluir otras? Estas y otras preguntas tendrán que ser abordadas seriamente. La experiencia en el ámbito inglés muestra que las estrategias de medición y evaluación no está exentas de fuertes críticas y que su impacto va más lejos.

Concluiremos señalando que las diferentes ideas expuestas sirven para visibilizar una transformación que ya está sucediendo y que afecta a la forma de conceptualizar la educación superior, sus funciones y sus relaciones. Aunque haya sistemas universitarios que aún estén lejos de parecerse al inglés, no son ajenos a este cambio y por ello deben de estar preparados para afrontarlo de forma crítica e informada. Se trata de una modernización que no negamos como necesaria, tanto a nivel europeo como español, pero que entendemos puede llevarse a cabo de forma que se minimicen algunos de sus aspectos más nocivos.

\section{Referencias}

Aramburuzabala, P., Hernández-Castilla, R., y Ángel-Uribe, I. C. (2013). Modelos y tendencias de la formación docente universitaria. Profesorado. Revista de Currículum y Formación de Profesorado, 17(3), 345-357.

Artess, J., Hooley, T. y Mellors-Bourne, R. (2017). Employability: A Review of the Literature 2012-2016. York: Higher Education Academy.

Bachan, R. (2017). Grade inflation in UK higher education. Studies in Higher Education, 428, $1580-1600$.

Ball, S. (2010). The teacher's soul and the terrors of performativity. Journal of Education Policy, 18(2), 215-228.

Ball, S. (2012). Global education Inc. London: Routledge.

Bárcena Orbe, F. (2019). La intimidad del estudio como forma de vida. Teoría de La Educación. Revista Interuniversitaria, 31(2), 41-67

Barnett, R. (2011). The Marketised university: defending the indefensible. En Molesworth, Nixon \& Scullion (Eds.). The marketisation of Higher Education and the Student as consumer (pp. 39-51). London: Routledge. 
Bates, E. y Kaye, L. (2014). 'I'd be expecting caviar in lectures': the impact of the new fee regime on undergraduate students' expectations of Higher Education. Higher Education, 67, 655-673.

Biesta, G. (2016). Good Education in an Age of Measurement: Ethics, Politics and Democracy. Oxon: Routledge.

Boden, R., y Nedeva, M. (2010). Employing discourse: Universities and graduate 'employability'. Journal of Education Policy, 25, 37-54.

Broecke, S. (2012). University rankings: Do they matter in the UK? Education Economics 23(2), 137-161.

Brown, W. (2015). Undoing the demos. New York: Zone books.

Brown, G., Wood, .M., Ogden, R.S. y Maltby, J. (2015). Do Student Evaluations of University Reflect Inaccurate Beliefs or Actual Experience? A Relative Rank Model. Journal of Behavioral Decision Making, 28(1), 14-26.

Browne, J. (2010). Securing a Sustainable Future for Higher Education: Report of the Independent Review of Higher Education Funding and Student Finance. London: Department for Business, Innovation and Skills.

Brunner, J. J. (2012). La Universidad: ¿Comunidad de mercado o posmoderna? Bordon, 64(3), 27-38.

Bryson, C. (2004). What about the Workers? Expansion of higher education and the transformation of academic work. Industrial Relations Journal, 35(1), 38-57.

Caroleo, F. E., Demidova, O., Marelli, E., y Signorelli, M. (2017). Young people and the labour market: A comparative perspective. London: Routledge.

Chubb, J., y Watermeyer, R. (2017) Artifice or integrity in the marketization of research impact? Investigating the moral economy of (pathways to) impact statements within research funding proposals in the UK and Australia. Studies in Higher Education, 42(12), 2360-2372.

Cilleros, R. y Betancor, G. (2014). 7. El movimiento estudiantil en la España del siglo XXI. Anuario del Conflicto Social, (4).

Collini, S. (2017). Speaking of Universities. London: Verso.

Collini, S. (2012). What are universities for? London: Penguin.

Cuenca, R. (2012). Sobre justicia social y su relación con la educación en tiempos de desigualdad. Revista Internacional de Educación para la Justicia Social (RIEJS), 1(1), 79-93.

De Faramiñan Gilbert, J. M. (2010). Against Bolonia. Revista de Estudios Jurídicos, 10, $1-11$.

Department for Education (DfE) (2017). Policy Paper: Teaching Excellence Framework. Lessons Learned. London: Department for Education. 
Department for Education and Skills (DfES) (2003). The future of Higher Education. Norwich: The Stationary Office.

Docherty, T. (2015). Universities at war. London: Sage.

Dussel, E. D. (2007). Política de la liberación: historia mundial y crítica. Madrid: Editorial Trotta.

Egido, I, (1995) Política educativa de la Unión Europea: principales etapas de desarrollo. Tendencias Pedagógicas, 2, 19-29.

Esteban-Bara, F. (2019). La universidad light: Un análisis de nuestra Formación universitaria. Barcelona: Paidós.

Feldman, Z. y Sandoval, M. (2018). Metric Power and the Academic Self: Neoliberalism, Knowledge and Resistance in the British University. TripleC, 16(1), 214-233.

Gautier, E. (2007). Educación de calidad. Comentarios a la nueva propuesta de OREALC/UNESCO. Revista Iberoamericana sobre Calidad, Eficacia y Cambio en Educación, 5(3), 29-35.

Gibbons, S., Neumayer, E. y Perkins, R. (2015). Student satisfaction, league tables and university applications: Evidence from Britain. Economics of Education Review, 48,148-164.

Giroux, H.A. (2014). Neoliberalism's War on Higher Education. Chicago: High Market Books.

García Ruiz, M. J. (2011). Impacto de la globalización en la universidad europea del siglo XXI The Impact of Globalisation in the European University of the 21st Century. Revista de Educación, 356, 509-529.

Glover, D., Law, S. y Youngman, A. (2002). Graduateness and Employability: student perceptions of the personal outcomes of university education. Research in PostCompulsory Education, 7(3), 293-306.

González-Serrano, M. D. (2011). Una aproximación a los aspectos positivos y negativos derivados de la puesta en marcha del Plan Bolonia en la Universidad Española. REJIE: Revista Jurídica de Investigación e Innovación Educativa, 4, 91-104.

Gumport, P. (2000). Academic restructuring: organizational change and institutional imperatives. Higher Education, 39(1), 67-91.

Gunn, A. (2018). Metrics and methodologies for measuring teaching quality in higher education: developing the Teaching Excellence Framework (TEF). Educational Review, 70 (2), 129-148.

Harvey, D. (2005). A brief history of Neoliberalism. Oxford: Oxford University Press.

Harvey, L. (2001). Defining and measuring employability. Quality in Higher Education, $7(2), 97-110$. 
Jones, G. (2010). Managing student expectations: The impact of top-up tuition fees. Perspectives, 14(2), 44-48.

Jongbloed, B. (2003). Marketisation in higher education, Clark's Triangle and the essential ingredients of markets. Higher Education Quarterly, 57(2), 110-135.

Knight, P. y Yorke, M. (2004). Learning, Curriculum and Employability in Higher Education. London: Routledge.

Kolsaker, A. (2008). Academic professionalism in the managerialist era: a study of English universities. Studies in Higher Education, 33(5), 513-525.

Masschelein, J. (2017). Some notes on the university as studium: a place of collective public study. En C. Ruitenberg (Ed.), Reconceptualizing study in educational discourse and practice (pp. 40-54). London: Routledge.

Maisuria, A. y Cole, M. (2017). The neoliberalization of higher education in England: An alternative is possible. Policy Futures in Education, 15(5), 602-619.

McGettigan, A. (2013). The great university gamble: money, markets and the future of higher education. London: Pluto Press.

Meyer, H-D.(2002) The new managerialism in education management: corporatization or organizational learning? Journal of Educational Administration, 4O(6), 534551.

Molesworth, M., Nixon, E. y Scullion, R. (2009). Having, being and higher education: the marketisation of the university and the transformation of the student into consumer. Teaching in Higher Education, 14(3), 277-287

Morrish, L. y Sauntson, H. (2013). 'Business-facing motors for economic development': an appraisal analysis of visions and values in the marketised UK university. Critical Discourse Studies, 10(1), 61-80.

Morrison, A. (2014). A class act? Lecturers' views on undergraduates' employability. British Journal of Sociology of Education, 35(4), 487-505.

Neary, M. y Saunders, G. (2016). Student as producer and the politics of abolition: Making a new form of dissident institution? Critical Education, 7(5), 1-23.

Neave, G. (2001). Educación superior: historia y política: Estudios comparativos sobre la universidad contemporánea. Barcelona: Gedisa Editorial.

Noonan, J. y Coral, M. (2015). The Tyranny of Work: Employability and the Neoliberal Assault on Education. Alternate Routes, 26, 51-73.

Olssen, M. (2016). Neoliberal competition in higher education today: Research, accountability and impact. British Journal of Sociology of Education, 37(1), 129148.

Ortega y Gasset, J. (1976). Misión de la Universidad y otros ensayos afines. Madrid: El arquero. 
Oxford Economics (2017). The economic impact of universities in 2014-15. Recuperado el 1 de Septiembre de 2018, de https://www.universitiesuk.ac.uk/policy-andanalysis/reports/Documents/2017/the-economic-impact-of-universities.pdf.

Pardo, J. L. (2013). El conocimiento líquido. Sobre la reforma de las universidades públicas. En J. Hernández, A. Delgado y X. Pericay (Ed.) La universidad cercada: testimonios de un naufragio (pp. 267-292). Barcelona: Anagrama.

Pickford, R. (2013). Leadership of the National Student Survey for enhancement. Quality Assurance in Education, 21 (4), 344-358.

Robbins, L. (1963). Higher education: report of the committee appointed by the Prime Minister under the Chairmanship of Lord Robbins Cmnd 2154. London: HMSO.

Salaburu, P., Haug, G. y Mora J-G. (2011). España y el proceso de Bolonia, un encuentro imprescindible. Madrid: Academia Europea de Ciencias y Artes.

Sanz-Fernández, F. (2006). La mercantilización de la educación como escenario mundial del Espacio Europeo de Educación Superior. Educación XX1, 9, 57-76.

Schildermans, H. (2019). Making a university. Introductory notes on an ecology of study practices. Leuven: KU Leuven (Unpublished doctoral dissertation).

Tejedor, F. J. y Jornet, J. M. . (2008). La evaluación del profesorado universitario en España. Revista Electrónica de Investigación Educativa, 10, 1-29.

Thiel, J. (2018). Student feedback apparatuses in higher education: an agential realist analysis. Discourse: Studies in the Cultural Politics of Education, pp.1-13.

Tight, M. (2013). Students: Customers, clients or pawns? Higher Education Policy, 26, 291-307.

Times Higher Education (THE) (2016, 20 Abril). NUS to encourage students to 'wreck' the TEF with NSS boycott.

Tomlinson, M. (2012). Graduate employability: A review of conceptual and empirical themes. Higher Education Policy, 25(4), 407-431.

Tomlinson, M. (2017). Student perceptions of Themselves as consumers of Higher Education. British Journal of Sociology of Education, 38(4), 450- 467.

Tomlinson, M. (2018). Conceptions of the value of higher education in a measured market. Higher Education, 75(4), 711-727.

Valle, J. M. (2004). La política educativa de la Unión Europea: fundamentos, evolución histórica y propuesta de un modelo para su análisis crítico. Revista Española de Educación Comparada, 10, 17-59.

Valle, J. M. (2012). La política educativa supranacional: un nuevo campo de conocimiento para abordar las políticas educativas en un mundo globalizado. Revista Española de Educación Comparada, 20, 109-144. 
Watermeyer, R. y Chubb, J. (2019). Evaluating 'impact'in the UK's Research Excelence Framework (REF): Liminality, looseness and new modalities of scholarly distinction. Studies in Higher Education, 44(9), 1554-1566.

Willetts, D. (2017). A University Education. Oxford: Oxford University Press.

Williams, J. (2013). Consuming Higher Education. London: Blomsbury.

Wilsdon, et al. (2015). The Metric Tide: Report of the Independent Review of the Role of Metrics in Research Assessment and Management. London: HEFC.

Yorke, M. (2006). Employability in Higher Education: What It Is - What It Is Not. York: Higher Education Academy. 